University of Nebraska - Lincoln

DigitalCommons@University of Nebraska - Lincoln

Robert Streubel Papers

Research Papers in Physics and Astronomy

$1-1-2015$

\title{
Magnetic chiral spin textures by imprinting
}

\author{
Robert Streubel \\ Leibniz-Institut für Festkörper- und Werkstoffforschung Dresden, streubel@unl.edu \\ F. Kronast \\ Helmholtz-Zentrum Berlin für Materialien und Energie (HZB) \\ U. Rössler \\ Institute for Theoretical Physics \\ O. G. Schmidt \\ Leibniz-Institut für Festkörper- und Werkstoffforschung Dresden \\ P. Fischer \\ Lawrence Berkeley National Laboratory
}

See next page for additional authors

Follow this and additional works at: https://digitalcommons.unl.edu/physicsstreubel

Part of the Atomic, Molecular and Optical Physics Commons, Condensed Matter Physics Commons, and the Other Physics Commons

Streubel, Robert; Kronast, F.; Rössler, U.; Schmidt, O. G.; Fischer, P.; and Makarov, D., "Magnetic chiral spin textures by imprinting" (2015). Robert Streubel Papers. 29.

https://digitalcommons.unl.edu/physicsstreubel/29

This Conference Proceeding is brought to you for free and open access by the Research Papers in Physics and Astronomy at DigitalCommons@University of Nebraska - Lincoln. It has been accepted for inclusion in Robert Streubel Papers by an authorized administrator of DigitalCommons@University of Nebraska - Lincoln. 


\section{Authors}

Robert Streubel, F. Kronast, U. Rössler, O. G. Schmidt, P. Fischer, and D. Makarov 


\title{
Magnetic chiral spin textures by imprinting
}

\author{
R. Streubel, ${ }^{1}$ F. Kronast, ${ }^{2}$ U. Rössler, ${ }^{3}$ \\ 0. G. Schmidt, ${ }^{1,4}$ P. Fischer, ${ }^{5,6}$ \& D. Makarov ${ }^{1}$ \\ 1 Institute for Integrative Nanosciences, IFW Dresden, Dresden, Germany \\ 2 Helmholtz-Zentrum Berlin für Materialien und Energie GmbH, Berlin, Germany \\ 3 Institute for Theoretical Physics, IFW Dresden, Dresden, Germany \\ 4 TU Chemnitz, Chemnitz, Germany \\ 5 Lawrence Berkeley National Laboratory, Berkeley, CA \\ 6 UC Santa Cruz, Santa Cruz, CA
}

The unique properties of non-trivial topological states, e.g. magnetic skyrmions [1] may path the way towards novel spintronic devices [2]. However, these spin textures have only been observed in special classes of materials possessing non-centrosymmetric crystal structure [1,3-6] and at low temperatures, which limits their application potential. We offer an alternate route to design synthetic magnetic heterostructures that resemble swirls, vortices or skyrmions with distinct topological charge densities at room temperature. By vertically stacking two magnetic nanopatterns with inand out-of-plane magnetization and tailoring the interlayer exchange coupling, non-collinear spin textures with tunable topological charge can be imprinted (Fig. 1a). We will present results on nanostructures consisting of soft-magnetic $40 \mathrm{~nm}$ thick Permalloy (Py, Ni80Fe20) and $5 \mathrm{~nm}$ thick Co/Pd multilayers. First we conduct systematic micromagnetic simulations of the magnetic spin texture in a

Published in 2015 IEEE International Magnetics Conference (INTERMAG).

Conference held 11-15 May 2015, Beijing, China; Session CG-05.

doi:10.1109/INTMAG.2015.7156875

Published by IEEE. Used by permission. 


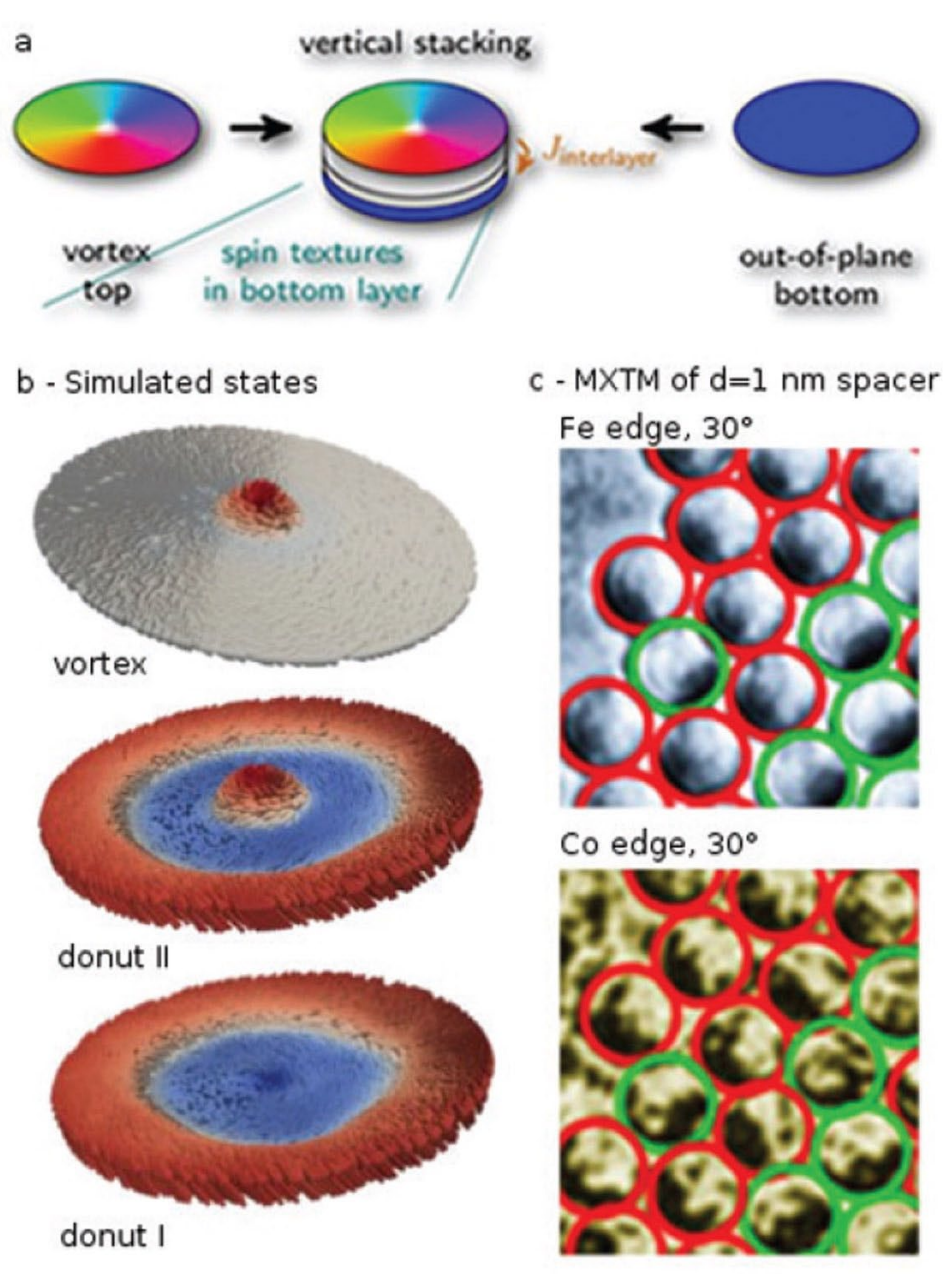

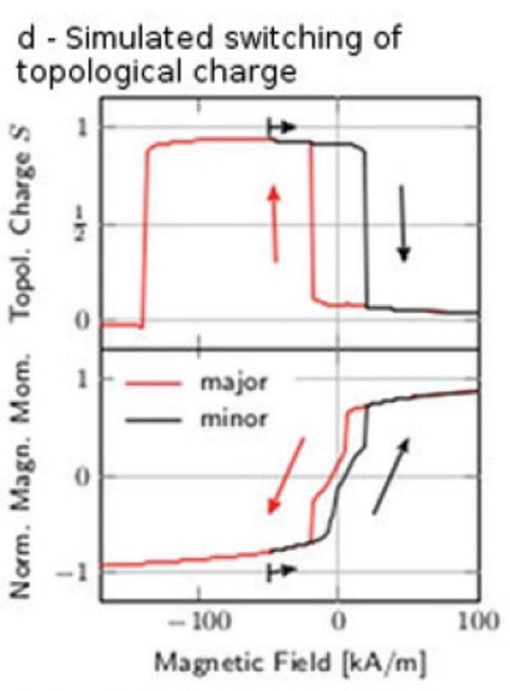

e - Line profile of out-of-plane magnetization

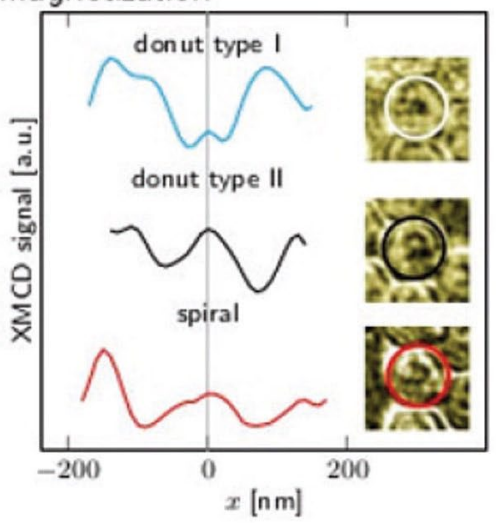

Figure 1

a) Conceptual approach: Vertically stacking of out-of-plane magnetized and magnetic vortex state imprints non-collinear spin textures into the originally out-ofplane magnetized film.

(b) Ensemble of remanent states obtained by micromagnetic simulations of nanopatterned disks.

(c) Experimental proof via MXTM of imprinting magnetic vortices into $\mathrm{Co} / \mathrm{Pd}$ multilayers with originally outof- plane anisotropy.

(d) Simulations show that an intermediate interlayer coupling strength allows for switching the topological charge of donut states at remanence and at room temperature by applying small magnetic fields.

(e) Line profile of the out-of-plane magnetization component for donut state I and II and magnetic spiral. 
disk-like heterostructure with a diameter of $400 \mathrm{~nm}$ where the interlayer exchange coupling strength between Py and Co/Pd sub-systems is varied from 0.1 to $2 \mathrm{~mJ} / \mathrm{m} 2$. In strongly coupled heterostructure, the magnetic state of the $\mathrm{Co} / \mathrm{Pd}$ is determined by the spin configuration in the Py disk resulting in the in-plane circulation of the magnetization (Figs. 1b,c). At an intermediate coupling strength, remanent donut states with a central vortex within the Co/Pd layers occur. Driving full or minor hysteresis loops allows to stabilize topologically distinct donut states with topological charges of about 1 and 0 . The magnetic configuration of the donut state set at remanence after saturating the sample is similar to those found in a disk with Dzyaloshinskii-Moriya interaction [7] hence suggesting that skyrmion-like structures can be experimentally realized in exchange coupled heterostructures. Owing to the larger switching field of the vortex core compared to the surrounding Co/Pd spins, a reversible switching between the two states is achieved. In this way, the topological charge can be switched in a digital manner at room temperature and at zero field (Fig. 1d). As our concept does not rely on vortex polarity switching in contrast to [8], much lower static magnetic fields are needed to manipulate the topological state of the material.

We have experimentally studied the magnetization reversal process and magnetic domain patterns in nanostructured [Co/Pd]/Pd/ Py stacks sputter deposited onto assemblies of non-magnetic silica spheres with a diameter of $500 \mathrm{~nm}$. The Pd spacer thickness is varied from 1 to $30 \mathrm{~nm}$. Evidence for the stabilization of non-collinear spin textures in the Co/Pd layers is provided by direct imaging of the magnetization patterns utilizing X-ray magnetic circular dichroism (XMCD) with high resolution magnetic soft X-ray transmission microcopy (MTXM) and X-ray photoemission electron microscopy (XPEEM). For a $1 \mathrm{~nm}$ spacer thickness, a vortex structure is observed (Fig. 1c). At $3 \mathrm{~nm}$ spacer thickness, an out-of-plane magnetization component appears in the Co/Pd sub-system. However, the circulation of the in-plane magnetization component still remains. For this sample, we clearly identify formation of different donut states as well as multi-domain states (Fig. 1e). The experimentally resolvable in-plane XMCD contrast in Co/Pd stack vanishes for the sample with $5 \mathrm{~nm}$ spacer thickness. The core of the imprinted vortex in the $\mathrm{Co} / \mathrm{Pd}$ multilayer is enlarged (60-110 nm) with respect to the Py vortex core due to the additional intrinsic out-of-plane anisotropy of $\mathrm{Co} / \mathrm{Pd}$. 
Modifications in both core size and out-of-plane magnetization components with respect to vortices in nanopatterned soft-magnetic materials induce distinct magnetization dynamics with time dependent gyrofrequencies. Insight into these complex magnetization dynamics will be given by comparing the experimental results with micromagnetic modeling. To conclude, we experimentally realize artificial non-collinear magnetic textures possessing distinct topological properties. The topological charges of these states can be manipulated by applying moderate magnetic fields at room temperature. Our concept provides an attractive and adaptable way to alter topological states, vortex core profiles and dynamic properties by tailoring the interlayer exchange coupling between the Py and Co/Pd layers.

\section{$\diamond \diamond \diamond \diamond \diamond \diamond$}

Supported by DFG (MA 5144/2-1) and ERC under European Union's Seventh Framework program (FP7/2007-2013)/ERC n.306277. PF acknowledges support from BES, MSED of the U.S. Department of Energy (DE-AC02-05- CH11231) and by Leading Foreign Research Institute Recruitment Program (2012K1A4A3053565) through the NRF of Korea funded by Ministry of Education, Science and Technology.

\section{References}

[1] U. K. Rößler, A. N. Bogdanov, and C. Pfleiderer, Nature (London) 442, 797 (2006).

[2] A. Fert, V. Cros, and J. Sampaio, Nat. Nano. 8, 152 (2013).

[3] X. Z. Yu, Y. Onose, N. Kanazawa, J. H. Park, J. H. Han, Y. Matsui, N. Nagaosa, and Y. Tokura, Nature (London) 465, 901 (2010).

[4] F. Jonietz, S. Mühlbauer, C. Pfleiderer, A. Neubauer, W. Münzer, A. Bauer, T. Adams, R. Georgii, P. Böni, R. A. Duine, K. Everschor, M. Garst, and A. Rosch, Science 330, 1648 (2010).

[5] S. Heinze, K. von Bergmann, M. Menzel, J. Brede, A. Kubetzka, R. Wiesendanger, G. Bihlmayer, and S. Blugel, Nat. Phys. 7, 713 (2011).

[6] N. Kanazawa, Y. Onose, T. Arima, D. Okuyama, K. Ohoyama, S. Wakimoto, K. Kakurai, S. Ishiwata, and Y. Tokura, Phys. Rev. Lett. 106, 156603 (2011).

[7] H. Du, W. Ning, M. Tian, and Y. Zhang, Phys. Rev. B 87, 014401 (2013).

[8] J. Li, A. Tan, K. Moon, A. Doran, M. Marcus, A. Young, E. Arenholz, S. Ma, R. Yang, C. Hwang, and Z. Qiu, Nat. Commun. 5, 4704 (2014). 